\title{
Metodologías activas en el área de inglés. Propuesta didáctica: The beach
}

DOI: $10.46932 / \mathrm{sfjdv2n1-037}$

Received in: November 1st, 2020

Accepted in: December 30th, 2020

\author{
Jose María Rabal Alonso \\ Profesor ISEN Centro Universitario \\ Institution: ISEN \\ Calle Campus Universitario, 12, 30100 Murcia \\ E-mail: josemaria.rabal@um.es \\ Andrea Ganga Cano \\ Graduada Educación Infantil \\ Institution: RG Formación \\ Calle Rosalía de Castro, 44, 30107 Murcia \\ Marta Bermejo Zárate \\ Graduada Educación Infantil \\ Institution: RG Formación \\ Calle Rosalía de Castro, 44, 30107 Murcia
}

\section{RESUMEN}

Este trabajo de investigación consiste en una secuencia de enseñanza dirigida a alumnos y alumnas de un aula de 5 años. La temática principal de este será la playa, pero incluida dentro del área de inglés. El objetivo más importante que queremos conseguir es potenciar el conocimiento de esta materia a través de un tema motivador e interesante para propio alumnado, por tanto, será de gran interés para ellos y la puesta en práctica les producirá un alto grado de motivación y les incitará a participar. Además, es un tópico bastante cercano y familiar para niños de edades tempranas, ya que es un medio de escape recurrente para las personas cuando les interesa deshacerse del estrés y la planificación rutinaria.

Para colmo, es un tema que nos ayudará a trabajar diversas partes del currículo del segundo ciclo de Educación Infantil, no solo el apartado de lengua extranjera, por lo que diríamos que es globalizador. La secuencia contará con seis sesiones y la evaluación de esta será la creación de una "final task" o tarea final donde quedarán reflejados todos los contenidos tratados en esta unidad.

Palabras clave: Organización escolar, didáctica, inglés, aprendizaje significativo, infantil.

\section{JUSTIFICACIÓN/INTRODUCCIÓN.}

Nuestra Unidad Didáctica “The beach” está diseñada para ser realizada por alumnos del Segundo Ciclo de Educación Infantil, en concreto para el alumnado de 5 años. No obstante, podría adaptarse a cualquier grupo de enseñanza de Infantil, reduciendo o ampliando el grado de dificultad de las actividades.

Para llevarla a cabo, siempre tendremos presente los conocimientos previos de los alumnos para ir introduciendo nuevos contenidos. El tema general de la Unidad Didáctica es la playa, ya que es un aspecto de la realidad próxima a los niños. La importancia del tema elegido es tal que se encuentra recogido en el $\mathrm{BOE}$ de $2^{\circ}$ Ciclo de Educación Infantil, concretamente en el área "Conocimiento del 
entorno".

Hemos intentado reflejar lo mejor posible este tema a través de diversos juegos que se pueden realizar en la playa, deportes y banderas que podemos encontrar. Hemos elegido este tema ya que creemos que es muy atractivo y motivador para ellos. Está relacionado con las vacaciones y por lo general a todos les suele gustar ir a la playa en este periodo del año.

La duración de esta Unidad Didáctica es de 6 sesiones, en las cuales los alumnos trabajarán diferentes aspectos, siendo siempre el alumno protagonista de su aprendizaje. Por último, señalar que conocemos la dificultad que presenta un idioma extranjero que únicamente se trabaja unas horas en concreto a la semana, por lo que iremos aumentando el ritmo y la dificultad, en función del manejo del idioma y de las sesiones adaptándonos a la realidad del aula.

\section{EXPLICACIÓN DE LA TAREA FINAL (FINAL TASK).}

La tarea final de esta Unidad Didáctica, se va a realizar con el objetivo de que los alumnos comprendan todos los conceptos, vocabulario y estructuras lingüísticas que se han trabajado durante toda la Unidad Didáctica.

La última sesión, que es la 6, los alumnos trabajarán libremente dejándoles desarrollar su creatividad y así dejar que nos sorprendan y deslumbren con todos los conocimientos que han adquirido durante las actividades de las sesiones anteriores. En esta sesión, los alumnos trabajarán libremente, la ayuda de la maestra solo se precisará en caso necesario.

Esta última actividad consiste en dividir la clase en varios grupos de alumnos, los cuales serán heterogéneos. La actividad que se va a llevar a cabo consiste en hacer un gran mural el cual cuando esté terminado se expondrá a toda la clase y se podrá quedar decorando el aula de infantil.

La maestra le proporcionará el material necesario a cada grupo y después de esto se sentará en su mesa a supervisar a los alumnos sin ayudarles en nada. Los alumnos dibujaran, pintarán, recortaran aquellos dibujos que hayan hecho y que les ha tocado para después ponerlos en el gran mural.

Habrá un grupo que se dedique a hacer la ropa que es adecuada para llevar a la playa. Otro grupo los objetos materiales con los que te puedes divertir en la arena o en el agua como son las raquetas, los cubos para hacer castillos de arena, una tabla de surf hinchable... Y otro grupo se dedicará a hacer las distintas banderas que nos podemos encontrar en la playa y que nos indican y advierten de si podemos o no bañarnos en la playa.

Una vez que han terminado todos los grupos de hacer sus correspondientes materiales, se levantará alumno por alumno y colocará su material en la zona del mural que le corresponda. Una vez terminado todo el mural, se mostrará a toda la clase completo y se colgará en alguna zona del aula. 
En esta actividad final, la profesora que no ha intervenido en ningún momento, ha sido una mera observadora, podrá saber si los alumnos han adquirido los conocimientos básicos de la Unidad Didáctica.

\section{OBJETIVOS DE APRENDIZAJE.}

Al terminar de trabajar con esta Unidad Didáctica, los alumnos deben haber alcanzado los siguientes resultados de aprendizaje:

- $\quad$ Capacidad para recordar conocimientos adquiridos anteriormente. (Actividad 2 -

\section{Sesión 1).}

- Aprender y practicar determinados patrones lingüísticos, además de aplicarlos correctamente.

(Todas las actividades - todas las sesiones).

- Saber utilizar y reproducir adecuadamente el vocabulario y las estructuras gramaticales trabajadas en la unidad didáctica (Todas las actividades - todas las sesiones).

- Conocer y nombrar en inglés los diferentes colores: rojo, verde, amarillo y blanco.

(Actividad 2 - Sesión 2).

- Reconocer la representación de las banderas asociándolas con sus colores correspondientes.

(Actividades 3 y 4 - Sesión 2, Actividad 2 - Sesión 4).

- Retener la información adquirida durante la lectura del "format". (Actividad 3 - Sesión 3).

- Saber utilizar posteriormente la información retenida durante la canción, el format o una explicación. (Actividad 5 - Sesión 3, Actividades 2 y 4 - Sesión 4).

- Responder correctamente a las preguntas sencillas que realiza el profesor. (Todas las actividades - todas las sesiones).

- Identificar el vocabulario relativo al tiempo meteorológico. (Actividad 1 - Sesiones 1, 2, 3, 4, 5 y 6, Actividad 3 - Sesión 4).

- Conocer y nombrar las diversas actividades y juegos que podemos realizar en la playa.

\section{(Actividad 5 - Sesión 3, Actividad 3 - Sesión 5).}

- Saber expresar con gestos y ademanes las emociones y sentimientos. (Todas las actividades - todas las sesiones).

- Construir y reconstruir textos de forma semi-autónoma con ayudas contextuales y lingüísticas, a través de información dada anteriormente. (Actividad 2 - Sesión 5).

- Reconocer y asociar todas las flashcards utilizadas durante la unidad didáctica. (Todas las actividades - todas las sesiones).

- Saber interactuar e interaccionar con los demás utilizando la lengua extranjera. (Todas las actividades - todas las sesiones). 
- Asimilar reglas de funcionamiento de diferentes juegos explicadas por el profesor.

(Actividad 3 - Sesión 1, Actividad 2 - Sesión 2, Actividad 3 - Sesión 5).

- Participación en todas las actividades propuestas durante el trascurso de la unidad didáctica.

(Todas las actividades - todas las sesiones).

- Recrear con adecuada temporalización y secuenciación una historia ya asimilada.

(Actividad 2 - Sesión 5).

- Comprender oralmente todo el contenido de la unidad didáctica. (Todas las actividades todas las sesiones).

- Reproducir adecuadamente los gestos durante canciones y cuentos. (Actividades primera y última - todas las sesiones, Actividad 3 - Sesión 2, Actividad 3 - Sesión 3).

- $\quad$ Conocer la ropa de uso típico en la playa. (Actividad 2 - Sesión 1).

- Dibujar, colorear y recortar con precisión diferentes figuras. (Tarea final - Sesión 6).

- $\quad$ Exponer con claridad el mural relativo a la tarea final. (Tarea final - Sesión 6).

- Prestar y mantener la atención durante explicaciones o clases teóricas. (Actividades 2 y 3 Sesión 3, Actividad 3 - Sesión 4, Actividad 2 - Sesión 6).

- Conocer diferentes tipos de playas. (Actividades 3 y 4 - Sesión 4).

- Cantar canciones llevando el ritmo y la entonación. (Actividades primera y última -

todas las sesiones, Actividad 3 -Sesión 3, Actividad 2 - Sesión 4).

\section{CONTENIDOS LINGÜÍSTICOS Y PROCESUALES DE LA UD SIGUIENDO EL CURRÍCULO DE LA REGIÓN DE MURCIA (6 AGOSTO 2008).}

Ahora detallaremos los contenidos lingüísticos y procesuales de nuestra Unidad Didáctica que aparecen en el Currículo de la Comunidad Autónoma de Murcia:

\section{Área 3: Lenguajes v Comunicación}

\section{Bloque 1: Lenguaje Verbal.}

- Escuchar, hablar y conversar.

\footnotetext{
* Comprensión de la idea global/información específica de mensajes orales en LE relacionado con situaciones habituales de aula tales como la narración de un cuento.

* Comprensión de la idea global/información específica de mensajes orales en LE relacionado con el tema de la playa.

* Comprensión y reacción a instrucciones en LE, asociadas a tareas usuales del aula tales como juegos habituales.
} 
* Comprensión y representación de una canción/cuento/poesía sobre la playa.

* Participación creativa en juegos lingüísticos para divertirse y aprender la lengua extranjera.

- Aproximación a la lengua escrita.

* Asociación de información oral a imágenes de colores y juegos.

- Acercamiento a la literatura.

* Escucha y comprensión de un cuento, relato, leyenda, poesía o adivinanza, etc. sobre la playa como fuente de placer y de aprendizaje.

* Dramatización del cuento/canción/poesía y disfrute e interés por expresarse con ayuda de recursos extralingüísticos.

- Conocimientos lingüísticos.

\begin{tabular}{|c|c|c|}
\hline Funciones & Modelos lingüísticos & Vocabulario \\
\hline $\begin{array}{l}\text { Preguntar de qué color son las } \\
\text { banderas referidas al entorno de la } \\
\text { playa. }\end{array}$ & What color is the flag? & \\
\hline $\begin{array}{l}\text { Responder de qué color son las } \\
\text { banderas referidas al entorno de la } \\
\text { playa. }\end{array}$ & $\begin{array}{l}\text { The flag is (red) I like/I don't like } \\
\text { We can (play with) }\end{array}$ & Colours: red, yellow, green, white \\
\hline Expresar gustos. & Yes, I do No, I don't & $\begin{array}{l}\text { Make sandcastle, ball, beach } \\
\text { rackets... }\end{array}$ \\
\hline Expresar sugerencias. & We can swim We can't swim & Play, eat, do, make... \\
\hline \begin{tabular}{llll} 
Responder & \multicolumn{2}{c|}{ adecuadamente } \\
preguntas & realizadas por la \\
profesora. & &
\end{tabular} & These are This is & \\
\hline $\begin{array}{l}\text { Expresar si se puede nadar o no, } \\
\text { según qué banderas haya en la } \\
\text { playa. }\end{array}$ & & 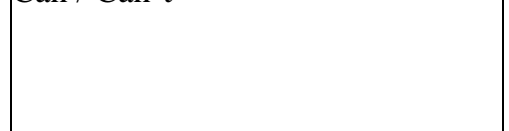 \\
\hline $\begin{array}{l}\text { Reconocer y nombrar diferente } \\
\text { vocabulario } \\
\text { relativo a la playa. }\end{array}$ & & $\begin{array}{l}\text { Fish, towel, swimsuit, beach } \\
\text { umbrella, sunglasses }\end{array}$ \\
\hline
\end{tabular}

\section{Bloque 2: Lenguaje audiovisual y TICS}

\footnotetext{
* Acercamiento a una película/dibujo animado, etc. Sobre Lucy y la playa que ayude a la comprensión de los contenidos de la LE.
} 
Bloque 3: lenguaje artístico.

- Expresión plástica.

* Expresión y comunicación de los contenidos de la LE relacionado con los juegos que realizamos en la playa a través del dibujo/de producciones plásticas.

- Expresión musical.

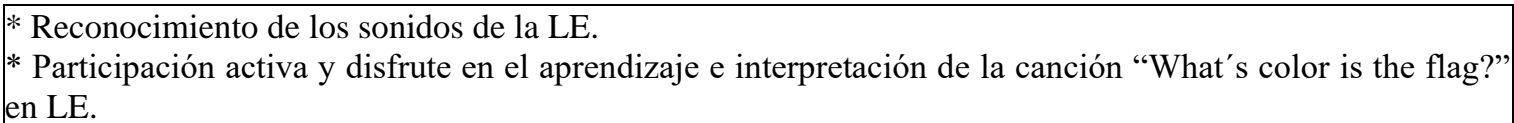

\section{Bloque 4: Lenguaje corporal.}

* Uso de gestos y movimientos para la comunicación en LE.
* Representación espontánea de personajes, hechos y situaciones en juegos simbólicos.
* Participación en actividades de dramatizaciones/bailes/juego simbólico/juegos de expresión corporal
relacionado con la playa en LE.

\section{DESARROLLO DE LA UNIDAD DIDÁCTICA (SESIONES).}

\section{SESIÓN 1}

- ACTIVIDAD 1: Warm-up routines.

- DURACIÓN: 5 minutos.

- OBJETIVOS: El objetivo de esta actividad es que los niños se preparen para la lección, fomentando la atención. Además, sirve para situar a los alumnos en un ambiente motivador.

- DESARROLlO: Pondremos a los alumnos en semicírculo en la asamblea, y cantaremos la canción de "Hello!", incitando a que los alumnos repitan los gestos de la misma. A continuación, realizaremos las rutinas. Para ello pediremos la ayuda del responsable del día.

- EJEMPLO DE INTERACCIÓN:

- Teacher: What is the weather like today?/ It's sunny?/ It's windy?/ It's cloudy?/ It's raining?/ It's snowing?

- Children: "Yes, it is/ No, it isn't"/ It's sunny/ It's raining, etc. 
- Teacher: What day is today?

- Children: Today is Monday (Tuesday, Wednesday, Thursday, Friday)

- ACTIVIDAD 2: Brainstorming.

- DURACIÓN: 15 minutos.

- OBJETIVOS: El objetivo de esta actividad es detectar conocimientos previos con el fin de generar asociaciones o generar hipótesis sobre el tema o contenidos a tratar a continuación. Implica una serie de contribuciones libres por parte de los alumnos.

- DESARROLlO: Pondremos a los alumnos en semicírculo en la asamblea, y cada uno va diciendo elementos que ellos conocen sobre la playa. Intentaremos que el alumno lo diga en inglés, pero si lo dice en español la profesora lo reforzará diciendo esa palabra en inglés. Después cada niño sale a la pizarra y dibuja el elemento que ha dicho.

\section{- EJEMPLO DE INTERACCIÓN:}

- Teacher: Hi children, we are going to make a brainstorming about the beach today. You have to say things about the beach.

- Children: Okey teacher!

- Teacher: Pepe, what's on the beach?

- Children: On the beach there are fish.

- Teacher: Okey, very good. Draw it in the sea.

- ACTIVIDAD 3: Games.

- DURACIÓN: 20 minutos.

- OBJETIVOS: El juego implica una competición entre los alumnos. El objetivo principal es practicar o aplicar determinados patrones lingüísticos a la vez que se introduce un elemento lúdico que les haga estar más pendientes del propósito de uso de la LE que de la forma lingüística.

- DESARROLLO: Dividimos la asamblea en dos partes con cinta aislante de colores. Una parte será el agua del mar y la otra parte la arena de la playa. A continuación, les enseñamos a los niños flashcards con los elementos que puede haber en la playa como una pelota, pez, castillo de arena, algas, etc. Ellos tienen que ir y posicionarse en la parte de la clase donde crean que pertenece ese elemento.

- EJEMPLO DE INTERACCIÓN: 
- Teacher: Hello children, we are going to play a game today.

- Children: Oh it is so funny.

- Teacher: Now I will show you a flashcard and you will run to water or to sand.

- Children: Okey teacher.

- ACTIVIDAD 4: Closing routines.

- DURACIÓN: 5 minutos.

- OBJETIVOS: El objetivo de esta actividad es que los niños se preparen para finalizar la lección de forma dinámica y motivadora.

- DESARROLLO: Pondremos a los alumnos en semicírculo en la asamblea, y cantaremos la canción de "Bye Bye Goodbye!", incitando a que los alumnos repitan los gestos de la misma. A continuación, la maestra se despedirá de los mismos de forma divertida.

\section{- EJEMPLO DE INTERACCIÓN:}

- Teacher: Now it's time to sing "Bye, bye!"

- Children: ¡Bien!

- Teacher and children: (Cantan la canción)

- Teacher: See you later alligator! (gesto de cocodrilo, estiramos los brazos hacia delante y ponemos una mano sobre otra dando palmadas) Bye Bye butterfly! (gesto de mariposa, entrelazamos los dos dedos pulgares y movemos el resto de los dedos hacia adelante y hacia detrás con las palmas de las manos situadas hacia dentro).

\section{SESIÓN 2}

- ACTIVIDAD 1: Warm-up routines.

- DURACIÓN: 5 minutos.

- OBJETIVOS: Desarrollados en la Sesión 1 - Actividad 1.

- DESARROLLO: Explicado en la Sesión 1 - Actividad 1.

- EJEMPLO DE INTERACCIÓN: Ejemplificado en la Sesión 1 - Actividad 1.

- ACTIVIDAD 2: Setting.

- DURACIÓN: 10 minutos.

- OBJETIVOS: Focalizar la atención de los alumnos sobre el tema que se va a tratar en la 
siguiente actividad o lección. Se trata de situar a los alumnos en un contexto que sea importante para la lección o actividad. Esto lo vamos a conseguir mediante un "Bingo de colores".

- DESARROLLO: Repartiremos a cada uno de los niños una cartulina a medio folio dividida en cuatro partes y cada parte será de un color. Puede haber algún color repetido. En el centro de la mesa habrá una caja con ceras de diferentes colores. La profesora comenzará con el bingo, irá diciendo los colores y los niños deberán repetir y coger la cera del color que la profesora dice y ponerla donde corresponda en la cartulina.

\section{- EJEMPLO DE INTERACCIÓN:}

- Teacher: Children, today we are going to play Bingo.

- Children: Oh! It's amazing!

- Teacher: Okey children. Now i'm going to show you the red color.

- Children: Red. (Los niños cogen el color rojo de la caja y lo ponen encima de la cartulina donde esté el color correspondiente.)

- ACTIVIDAD 3: Song presentation.

- DURACIÓN: 15 minutos.

- OBJETIVOS: El objetivo de esta actividad es que los niños aprendan distinto vocabulario y estructuras gramaticales a través de una canción que tiene que ver con el tema de la Unidad Didáctica: "La Playa".

- DESARROLLO: Pondremos a los alumnos en semicírculo en la asamblea de pie, y cantaremos la canción, incitando a que los alumnos repitan y entiendan la letra de ésta.

\section{- EJEMPLO DE INTERACCIÓN:}

- Teacher: Children, do you like songs?

- Children: Yes! Yes!

- Teacher: Ok! So now, let's sing a new song.

- CONTENIDOS LINGÜÍSTICOS:

\begin{tabular}{|l|l|l|}
\hline \multicolumn{1}{|c|}{ Funciones } & \multicolumn{1}{c|}{ Modelos lingüísticos } & \multicolumn{1}{c|}{ Vocabulario } \\
\hline $\begin{array}{l}\text { Preguntar de qué color son las } \\
\text { banderas referidas al entorno de } \\
\text { la playa. }\end{array}$ & What color is the flag? & \\
$\begin{array}{l}\text { Responder de qué color son las } \\
\text { banderas referidas al entorno de } \\
\text { la playa. }\end{array}$ & The flag is (red) & $\begin{array}{l}\text { Colours: red, yellow, green, } \\
\text { white }\end{array}$ \\
\hline
\end{tabular}


Expresar si se puede nadar o no, según qué banderas haya en la playa.

\begin{tabular}{|l|l|}
\hline We can swim & Can / Can't \\
We can't swim &
\end{tabular}

- ACTIVIDAD 4: Recognition.

- DURACIÓN: 10 minutos.

- OBJETIVOS: Comprobar que los alumnos reconocen lo que se ha trabajado en el format.

- DESARROLLO: La actividad consiste en que colocamos las tres flashcards de la pelota, las palas y los castillos de arena en distintos espacios del aula. La maestra dirá un juego y los niños deberán correr por el aula e ir a la zona de la flashcard dicha y señalarla gritando el nombre de la flashcard.

\section{- EJEMPLO DE INTERACCIÓN:}

- Teacher: Where's the sandcastle? (Alzando la voz en la palabra del juego al que tienen que ir)

\section{Children: Sandcastle!}

- ACTIVIDAD 5: Drill.

- DURACIÓN: 10 minutos.

- OBJETIVOS: Comprobar que los alumnos han afianzado los conocimientos enseñados sobre los gustos.

- DESARROLLO: La actividad consiste en que con los alumnos sentados en la asamblea deberán ir diciendo de uno en uno qué juego de los tres aprendidos que se pueden hacer en la playa les gusta y cual no les gusta. Las flashcards estarían colocadas en la pizarra de forma que los alumnos puedan visualizarlas.

- EJEMPLO DE INTERACCIÓN:

- Teacher: Which one do you like the most?

Children: I like the ball.

\section{SESIÓN 3}

- ACTIVIDAD 1: Warm-up routines.

- DURACIÓN: 5 minutos.

- OBJETIVOS: Desarrollados en la Sesión 1 - Actividad 1.

- DESARROLLO: Explicado en la Sesión 1- Actividad 1.

- EJEMPLO DE INTERACCIÓN: Ejemplificado en la Sesión 1 - Actividad 1. 
- ACTIVIDAD 2: Setting.

- DURACIÓN: 10 minutos.

- OBJETIVOS: Captar la atención de los niños y centrarlos en el tema que se va a trabajar en la siguiente actividad, así como contextualizar la primera narración del cuento "Lucy at the beach".

- DESARROLLO: La actividad consiste en que presentamos a Lucy utilizando una marioneta, para ello, el profesor captará la atención de los niños diciéndoles que va a contarles una nueva historia y les presentará a la protagonista de la misma: Lucy.

- EJEMPLO DE INTERACCIÓN:

- Teacher: I'm going to tell you a story about a girl and her holidays.

This is Lucy.

Hello Lucy!

- Children: Hello Lucy!

- ACTIVIDAD 3: Story presentation.

- DURACIÓN: 15 minutos.

- OBJETIVOS: El objetivo de esta actividad es que los niños aprendan distinto vocabulario y estructuras gramaticales a través de un cuento que tiene que ver con el tema de la Unidad Didáctica: "La Playa".

- DESARROLLO: Pondremos a los alumnos en semicírculo en la asamblea sentados, y les contaremos la historia ayudándonos de flashcards, para que así sea más sencillo comprender el format.

- EJEMPLO DE INTERACCIÓN:

- Teacher: Children, do you like stories?

- Children: Yes! Yes!

- Teacher: Ok! So now, let's tell a new story about a girl and her holidays.

- Teacher and children: Ok. One, two, three, look at me. One, two, three, listen to me. One, two, three, silence, please.

- CONTENIDOS LINGÜÍSTICOS:

\begin{tabular}{|l|l|l|}
\hline Funciones & Modelos linguiísticos & Vocabulario \\
\hline Expresar sugerencias & $\begin{array}{l}\text { We can play with (a } \\
\text { ball) }\end{array}$ & $\begin{array}{l}\text { Ball, beach } \\
\text { rackets and } \\
\text { sandcastle. }\end{array}$ \\
Expresar gustos & & \\
\hline
\end{tabular}




\begin{tabular}{|l|l|l|}
\hline & We & \\
don't & \\
like & \\
We & \\
& like! & \\
\hline
\end{tabular}

- ACTIVIDAD 4: Recognition.

- DURACIÓN: 10 minutos.

- OBJETIVOS: Comprobar que los alumnos reconocen lo que se ha trabajado en el format.

- DESARROLLO: La actividad consiste en que colocamos las tres flashcards de la pelota, las palas y los castillos de arena en distintos espacios del aula. La maestra dirá un juego y los niños deberán correr por el aula e ir a la zona de la flashcard dicha y señalarla gritando el nombre de la flashcard.

\section{- EJEMPLO DE INTERACCIÓN:}

- Teacher: Where's the sandcastle? (Alzando la voz en la palabra del juego al que tienen que ir)

Children: Sandcastle!

- ACTIVIDAD 5: Drill.

- DURACIÓN: 10 minutos.

- OBJETIVOS: Comprobar que los alumnos han afianzado los conocimientos enseñados sobre los gustos.

- DESARROLLO: La actividad consiste en que con los alumnos sentados en la asamblea deberán ir diciendo de uno en uno qué juego de los tres aprendidos que se pueden hacer en la playa les gusta y cual no les gusta. Las flashcards estarían colocadas en la pizarra de forma que los alumnos puedan visualizarlas.

- EJEMPLO DE INTERACCIÓN:

- Teacher: Which one do you like the most?

Children: I like the ball.

- ACTIVIDAD 5: Closing routines.

- DURACIÓN: 5 minutos.

- OBJETIVOS: Desarrollados en la Sesión 1 - Actividad 4.

- DESARROLLO: Explicado en la Sesión 1 - Actividad 4. 
EJEMPLO DE INTERACCIÓN: Ejemplificado en la Sesión 1 - Actividad 4.

\section{SESIÓN 4}

- ACTIVIDAD 1: Warm-up routines.

- DURACIÓN: 5 minutos.

- OBJETIVOS: Desarrollados en la Sesión 1 - Actividad 1.

- DESARROLLO: Explicado en la Sesión 1 - Actividad 1.

EJEMPLO DE INTERACCIÓN: Ejemplificado en la Sesión 1 - Actividad 1

- ACTIVIDAD 2: Song recitation.

- DURACIÓN: 10 minutos.

- OBJETIVOS: El objetivo de esta actividad es que los niños repasen el vocabulario y estructuras gramaticales a través de la canción anteriormente cantada, que tiene que ver con el tema de la Unidad Didáctica: “La Playa”. Implica la memorización y "reproducción oral".

- DESARROLlO: Pondremos a los alumnos en semicírculo en la asamblea de pie, y cantaremos la canción, animando a que los alumnos repitan y recuerden la letra de ésta.

- EJEMPLO DE INTERACCIÓN:

- Teacher: Children, do you remember the song "What color is the flag"?

- Children: Yes! Yes!

- Teacher: Ok! So now, let's sing the song.

- ACTIVIDAD 3: Content explanation.

- DURACIÓN: 15 minutos.

- OBJETIVOS: EI objetivo de esta actividad es la explicación de contenidos conceptuales o temáticos a los niños. Esta explicación es a nivel sociocultural.

- DESARROLlO: Les vamos a presentar el mapa de Reino Unido y le vamos a rodear el contorno del país. A continuación, con los niños sentados en la asamblea se les explicará el tiempo meteorológico que hace en Reino Unido, contándoles el mal tiempo que siempre suele hacer y comparándolo con el tiempo en España.

- EJEMPLO DE INTERACCIÓN: 
- Teacher: Hello children, today I am going to explain the weather of United Kingdom.

- Children: Perfect teacher.

- Teacher: In United Kingdom always it's bad weather and it's always raining.

- ACTIVIDAD 4: Checking.

- DURACIÓN: 10 minutos.

- OBJETIVOS: El objetivo es la comprobación por parte del profesor que recuerdan los alumnos sobre la información con la que han trabajado en la nueva actividad previa o comprobar los resultados obtenidos en dicha actividad.

- DESARROLLO: Se sentarán todos los niños en la asamblea, y en la pizarra digital se les mostrarán a los niños imágenes de playas con el tiempo meteorológico diferente. Una nevando, una lloviendo, haciendo sol, etc. La profesora irá nombrando a los niños y ellos deberán decir a que país pertenece ese tiempo meteorológico, si pertenece a Reino Unido o a España.

- EJEMPLO DE INTERACCIÓN:

- Teacher: Children, today let's differentiate the weather.

- Children: Okey teacher.

- Teacher: This photo belong to...

- Children: Belong to United Kindong.

- Teacher: This photo belong to...

- Children: Belong to Spain.

- ACTIVIDAD 5: Closing routines.

- DURACIÓN: 5 minutos

- OBJETIVOS: Desarrollados en la Sesión 1 - Actividad 4.

- DESARROLlO: Por motivo de su buen comportamiento cantaremos una canción donde los niños tienen que hacer una serie de actividades y las niñas otras. Dividiremos la clase en chicos y chicas y pondremos la canción.

- EJEMPLO DE INTERACCIÓN:

- Teacher: Today I am very happy because have you done the activities very well and we are going to dance the song "Girls and boys".

- Children: Bien!! 
- Teacher: Now the girls here and the boys there. Let's dance!

\section{SESIÓN 5}

ACTIVIDAD 1: Warm-up routines.

DURACIÓN: 5 minutos.

OBJETIVOS: Desarrollados en la Sesión 1 - Actividad 1.

DESARROLLO: Cantaremos la canción de "Hello!", como todos los días, desarrollada en la Sesión 1 - Actividad 1 y en vez de hacer las rutinas habituales, cantaremos el cumpleaños feliz a Marta, por motivo de su cumpleaños. Para ello pedimos a Marta que se levante y se ponga al lado de la profesora y todos juntos le cantaremos la canción de “Happy Birthday!".

\section{EJEMPLO DE INTERACCIÓN:}

- Teacher: Today is Marta's Birthday! Do you want to sing "Happy Birthday?

- Children: Yes!

- Teacher: Let's go!

- Children and teacher: "Happy birthday to you, happy birthday to you, happy birthday little Marta, Happy birthday to you!"

- Teacher: Happy birthday Marta! Everybody: "Happy birthday Marta!"

- Children: "Happy birthday Marta!"

- ACTIVIDAD 2: Cued narrative.

- DURACIÓN: 15 minutos.

- OBJETIVOS: El objetivo es que los alumnos construyan textos de forma semi- autónoma, ya que se les proporcionan ayudas contextuales y lingüísticas. Esto lo vamos a conseguir mediante la reconstrucción de la "Story Presentation" entre el alumno y el profesor con flashcard.

- DESARROLLO: Vamos a reconstruir la historia que contamos en la sesión 3 con la ayuda de los niños, y mediante flashcards para así ayudarles a recordar los juegos que aparecían durante el cuento. A demás les servirá para hacer la siguiente actividad.

- EJEMPLO DE INTERACCIÓN:

- Teacher: Hello children. Today I am going to tell the story about Lucy again. But now, I 
need your help, because I have forgot some things.

- Children: Okey teacher. We'll help you.

- Teacher: Once upon a time there was a girl called...

- Children: Lucy!

- Teacher: Who wanted to go the sea because se had never been to...

- Children: The beach!

- ACTIVIDAD 3: Games.

- DURACIÓN: 10 minutos.

- OBJETIVOS: Identificar el vocabulario de la playa trabajado anteriormente a través de un juego donde el alumno mostrará su comprensión con respuestas no lingüísticas.

- DESARROLlO: Jugaremos al Twister pero en vez de con colores, la alfombra llevará dibujos del vocabulario trabajado. Para ello aparecerán imágenes de castillos de arena en la playa, pelotas y raquetas. La profesora dirá una de esas actividades que se pueden hacer en la playa y los niños tendrán que ir a tocarlo con la mano o con el pie, hasta que se vayan cayendo.

- EJEMPLO DE INTERACCIÓN:

- Teacher: We are going to play Twister!

- Children: Good! Great!

- Teacher: Put your hand on "ball"/ put your leg on "beach racket"

- ACTIVIDAD 4: Checking.

- DURACIÓN: 10 minutos.

- OBJETIVOS: Comprobar por parte del profesor de que los alumnos recuerdan la información con la que han trabajado en una nueva actividad previa. Lo conseguiremos mediante una actividad en la que la profesora les hará preguntas a los alumnos para comprobar si saben las estructuras lingüísticas.

- DESARROLlO: La llevaremos a cabo después de un juego llamado "Twister". Sentaremos a los alumnos en la asamblea en semicírculo. La profesora les preguntara a los alumnos si les gustan los juegos que se han presentado en la "Story Presentation". Los alumnos contestaran con la estructura lingüística: Me gusta/No me gusta.

- EJEMPLO DE INTERACCIÓN: 
- Teacher: Hello children, today we will remember the games that appear in the story presentation.

- Children: Okey teacher!

- Teacher: Juan, do you like make sandcastle?

- Children: Yes, I like make sandcastle.

- Teacher: María, do you like play rackets?

- Children: No, I don’t like play rackets.

- ACTIVIDAD 5: Closing routines.

- DURACIÓN: 5 minutos.

- OBJETIVOS: Desarrollados en la Sesión 1 - Actividad 4.

- DESARROLLO: Explicado en la Sesión 1 - Actividad 4.

- EJEMPLO DE INTERACCIÓN: Ejemplificado en la Sesión 1 - Actividad 4.

\section{SESIÓN 6}

- ACTIVIDAD 1: Warm-up routines.

- DURACIÓN: 5 minutos.

- OBJETIVOS: Desarrollados en la Sesión 1 - Actividad 1.

- DESARROLLO: Explicado en la Sesión 1 - Actividad 1.

- EJEMPLO DE INTERACCIÓN: Ejemplificado en la Sesión 1 - Actividad 1.

- ACTIVIDAD 2: Organization.

- DURACIÓN: 5 minutos.

- OBJETIVOS: El profesor describe las reglas del funcionamiento de una actividad que se va a realizar a continuación. Incluye una explicación detallada, y con un discurso extendido, de los procedimientos generales para la interacción y la realización de las actividades. Los alumnos ayudados por el contexto y los gestos del profesor, entienden el contenido del discurso de este.

- DESARROLLO: Los alumnos se sientan en la asamblea y escuchan atentos la explicación de la maestra. La profesora les explica detalladamente a todos los alumnos como se va a realizar la tarea final que se va a hacer a continuación.

- EJEMPLO DE INTERACCIÓN:

- Teacher: Hello children, sit down in the assembly. 
- Children: Okey teacher.

- Teacher: Listen carefully that I am going to explain to you the following activity. It's very funny.

- Children: Oh, it's amazing.

- ACTIVIDAD 3: Final Task.

- DURACIÓN: 25 minutos.

- OBJETIVOS: Los objetivos de esta actividad final serán: conocer los elementos que se pueden llevar a la playa, como es la ropa que deben llevar las personas, así como todos los objetos que puedes utilizar en ella como las raquetas, pelotas hinchables, etc. Además, los niños conocerán los seres vivos que viven en él y también el tiempo meteorológico que hace y con el que si pueden bañarse en la playa.

- DESARROLLO: Se va a crear un gran mural sobre la playa por grupos. La clase se dividirá en grupos, valen los grupos en los que los niños trabajan normalmente en clase. Después se expondrá el mural realizado a toda la clase. Nuestra función como profesora será ofrecerle todo aquel material para que ellos realicen el mural. Cada grupo se encargará de hacer una parte para ese mural. Unos los juegos que puedes hacer en la playa, otros los animales que viven en el mar. Y finalmente se unirá en un gran mural el cual se expondrá para toda la clase.

- EJEMPLO DE INTERACCIÓN:

- Teacher: Hi children! Today finally this activity. That's why we are going to do a great final activity.

- Children: Okey teacher, we have learned a lot.

- Teacher: Perfect, now we are going to make a great mural about the beach.

- Children: Oh, it's exciting!

- Teacher: Okey, come on!

- ACTIVIDAD 4: Self-evaluation.

- DURACIÓN: 5 minutos.

- OBJETIVOS: Los objetivos de esta actividad son que los niños se evalúen a sí mismos para comprobar que han adquirido todos los conocimientos necesarios sobre esta unidad didáctica. Además de su capacidad, así como de la calidad del trabajo realizado.

- DESARROLLO: Los niños al realizar los materiales en grupo para el gran mural, y acto 
seguido exponerlo para toda la clase, si la profesora le felicita y reconoce su trabajo bien hecho, sabrá que todo lo que ha trabajado ha servido para adquirir los conocimientos sobre este medio natural. La profesora, pondrá una pegatina verde a todo aquel alumno que realice su tarea correctamente. De este modo el niño se autoevalúa y reflexiona si sabe o no sabe hacer lo que se le ha enseñado en las últimas seis sesiones.

- EJEMPLO DE INTERACCIÓN:

- Teacher: You are ready to show the mural?

- Children: Yes teacher.

(Los niños muestran sus materiales terminados para pegarlos en el gran mural)

- Teacher: Oh it's very beautiful, children. Are so good.

(La profesora obsequia a cada alumno con la pegatina verde por hacer bien el trabajo).

- Children: We did it.

- ACTIVIDAD 5: Closing routines.

- DURACIÓN: 5 minutos.

- OBJETIVOS: Desarrollados en la Sesión 1 - Actividad 4.

- DESARROLLO: Explicado en la Sesión 1 - Actividad 4.

- EJEMPLO DE INTERACCIÓN: Ejemplificado en la Sesión 1 - Actividad 4.

\section{EVALUACIÓN.}

En cuanto a la evaluación podemos decir que se van a llevar a cabo dos, es decir, una evaluación continua y una evaluación final. Sin embargo, el fin de ambas es comprobar si los objetivos previstos en la Unidad Didáctica han sido alcanzados.

En cuanto a la evaluación continua, podemos decir que vamos a estar atentas durante la realización de cada una de las actividades, llevadas a cabo en las sesiones 1, 2, 3, 4 y 5 para comprobar si se cumplen los criterios que se recogen en la siguiente tabla:

\begin{tabular}{|l|l|l|}
\hline Criterios a evaluar & Sí & No \\
\hline Respetar el turno de palabra & & \\
\hline Escuchar atentamente, tanto a la maestra como a los compañeros. & & \\
\hline Participa en las actividades. & & \\
\hline Respeta a todos y ayuda a los compañeros. & & \\
\hline Muestra interés por la tradición inglesa y sus características. & & \\
\hline A la hora de recoger colabora. & & \\
\hline
\end{tabular}

En cuanto a la evaluación final, ésta se va a llevar a cabo durante la realización de la tarea final. 
Aquí podremos comprobar si los niños han adquirido todos y cada uno de los conocimientos que hemos ido incorporando en cada una de las sesiones y en cada una de las actividades. Va a estar basada en los siguientes criterios:

\begin{tabular}{|l|l|l|}
\hline Criterios a evaluar & Sí & No \\
\hline \hline Reconoce y utiliza adecuadamente can y can't. & & \\
\hline Sabe utilizar las expresiones I like/I don't like. & & \\
\hline Comprende el vocabulario trabajado en la Unidad Didáctica. & & \\
\hline Es capaz de reproducir dicho vocabulario. & & \\
\hline Identifica las banderas de la playa. & & \\
\hline Comprende el cuento incluido en la Unidad Didáctica. & & \\
\hline Comprende la canción “What color is the flag”. & & \\
\hline
\end{tabular}

En definitiva, ambas evaluaciones se van a llevar de manera directa y a través de la observación de los niños a la hora de realizar las actividades, para poder comprobar el aprendizaje de cada uno de ellos, por lo que será individualizada, y así comprobar si han alcanzado el nivel deseado. 


\section{REFERENCIAS}

Bouso, J. L. M. (2012). Didáctica de inglés en educación infantil. Ideaspropias Editorial SL.

López, B. R. (2004). Técnicas metodológicas empleadas en la enseñanza del inglés en Educación Infantil. Estudio de caso. Didáctica. Lengua y Literatura, 16, 151-161.

Fonseca Mora, M. D. C., \& Martín-Pulido, S. (2015). Enseñar inglés en educación infantil: materiales y recursos. Tonos digital, $n^{\circ} 29,2015$. 